\title{
Caractérisation multi-échelle d'alliages nickel - titane utilisés pour des limes endodontiques
}

\author{
N. Serres ${ }^{1, a}$, N. Bahlouli², J. Hemmerlé ${ }^{3}$, C. Wagner-Kocher ${ }^{2}$, P. de Magalhaes Correia ${ }^{2}$, \\ R. Fabre ${ }^{3}$, L. Jordan ${ }^{4}$, Y. Haikel ${ }^{5}$ et F. Feugeas ${ }^{1}$ \\ ${ }^{1}$ ICube, INSA de Strasbourg, CNRS, 24 boulevard de la Victoire, 67084 Strasbourg Cedex, France \\ 2 ICube, Université de Strasbourg, CNRS, 2 rue Boussingault, 67000 Strasbourg, France \\ 3 INSERM, UMR 1121, unité Biomatériaux et Bioingénierie, Université de Strasbourg, France \\ ${ }^{4}$ Université Paris-Diderot, UFR d'Odontologie, Service d'Odontologie Hôpital Rothschild, Groupe de Métallurgie \\ Structurale UMR7045 - Chimie Paris Tech, France \\ ${ }^{5}$ Département d'Odontologie Conservatrice - Endodontie, Faculté de Chirurgie Dentaire, Strasbourg, France
}

\begin{abstract}
Résumé. L'analyse des comportements mécaniques, biologiques et physiques aux interfaces de biomatériaux médicaux ayant vocation à évoluer dans la sphère orale est particulièrement importante. En effet, des influences cliniques diverses, en termes de vieillissement des matériaux et de protection des éléments dentaires et péri-dentaires peuvent être constatées. Les alliages à mémoire de forme Nickel Titane sont couramment utilisés dans la fabrication d'instruments d'odontologie. Dans cette étude, deux systèmes de limes endodontiques ont été étudiés. Bien qu'ayant des compositions chimiques similaires, leurs structures différentes conduisent empiriquement à des modes de rupture distincts. En effet, certaines limes peuvent être utilisées plusieurs fois, alors que d'autres limes provenant d'un fournisseur différent peuvent casser dès la première utilisation, sans prévention possible. Ce travail cherche à comprendre cette différence à travers une étude couplée mécanique/métallurgique afin de corréler le comportement en traction et en fatigue avec l'état de surface des limes, corrélés à leurs procédés d'élaboration. L'étude métallurgique a d'abord été focalisée sur une comparaison des états de surface, avant et après des traitements de mordançage et/ou de polissage électrolytique (Fig. 1). L'étude mécanique a débuté par l'identification expérimentale des matériaux utilisés pour la fabrication de ces deux types de limes dentaires. Ces matériaux ont été fournis sous forme de fils. La littérature met en évidence la sensibilité du comportement de ces alliages à la vitesse de déformation. Deux vitesses de sollicitations sur trois fils de diamètre $0,6 \mathrm{~mm} ; 0,8 \mathrm{~mm}$ et $1 \mathrm{~mm}$ ont été testés. Des essais de traction (Fig. 2) et de fatigue (Fig. 3) ont été réalisés sur ces trois diamètres. Les faciès de rupture avant et après essais ont aussi été analysés.
\end{abstract}
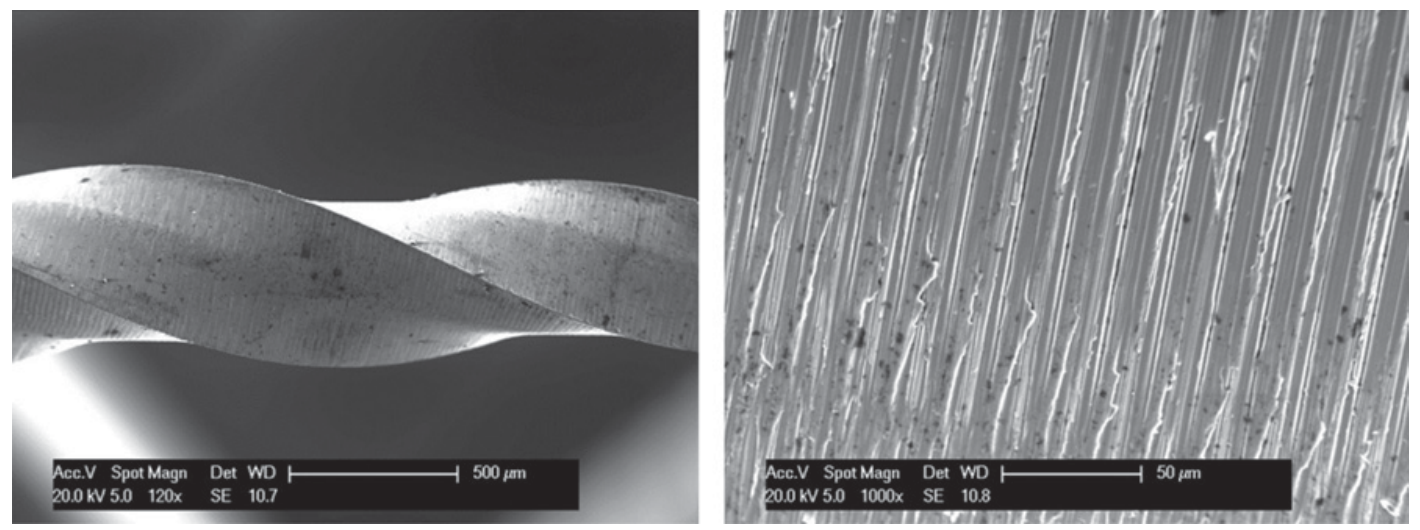

Figure 1. Observations MEB de limes endodontiques mordançées.

\footnotetext{
a e-mail : nicolas.serres@insa-strasbourg.fr
} 
MATEC Web of Conferences

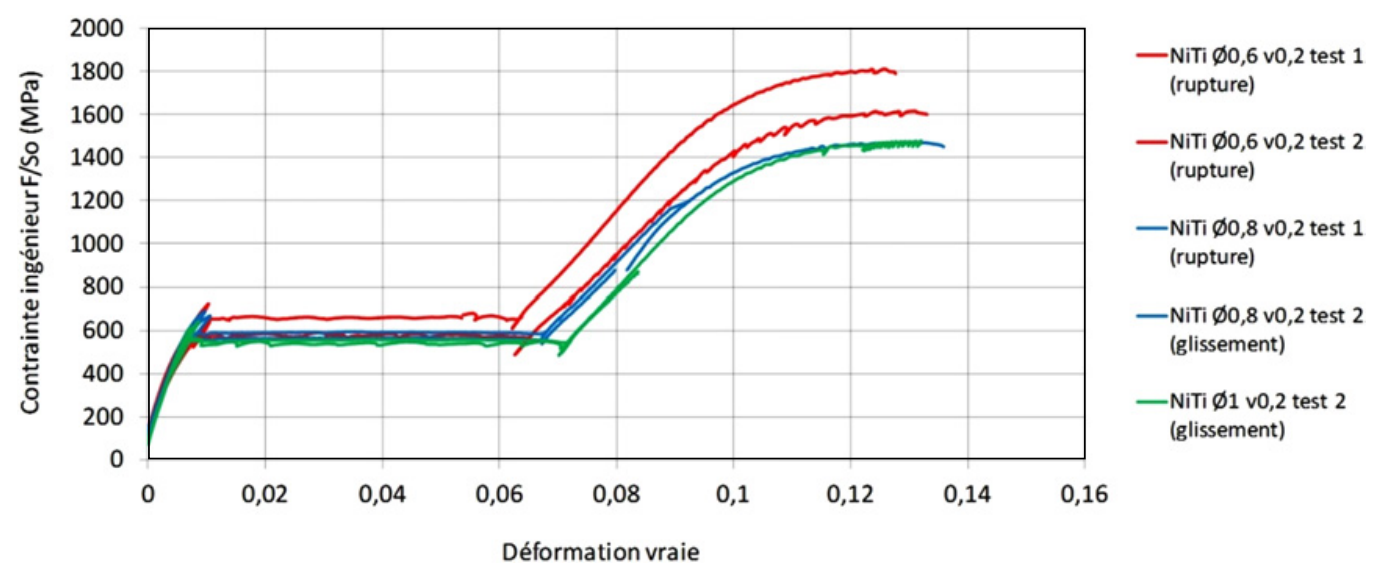

Figure 2. Essai de traction sur Fil NiTi (Vitesse de la traverse : $0,8 \mathrm{~mm} / \mathrm{min}$ ).

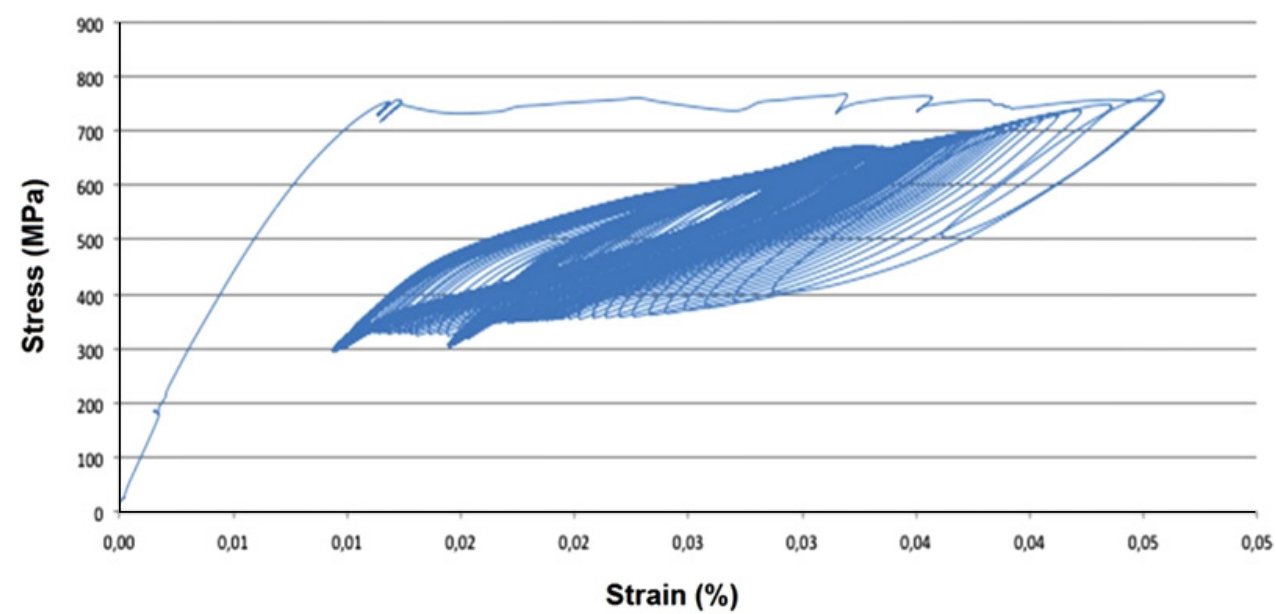

Figure 3. Essai de fatigue sur fil NiTi (Vitesse de la traverse : $120 \mathrm{~mm} / \mathrm{min}$ jusqu'à $\varepsilon=4,5 \%$ suivi d'un chargement de fatigue sinusoïdale 500 cycles à $5 \mathrm{~Hz}$ - amplitude : 0,4 mm). 